\title{
Myofascial Pain Dysfunction Syndrome: A Revisit
}

\author{
Dr. Rhea reji ${ }^{1}$, Dr. Vasavi krishnamurthy ${ }^{2}$, Dr. Mandavi garud ${ }^{3}$ \\ ${ }^{l}$ (department of oral medicine and maxillofacial radiology/ d.y. Patil university, school of dentistry, navi \\ mumbai, india) \\ ${ }^{2}$ (department of oral medicine and maxillofacial radiology/ d.y. Patil university, school of dentistry, navi \\ mumbai, india) \\ ${ }^{3}$ (department of oral medicine and maxillofacial radiology/ d.y. Patil university, school of dentistry, navi \\ mumbai, india)
}

\begin{abstract}
Myofascial Pain Dysfucntion Syndrome is a heterogeneous group of signs and symptoms that affect the jaw joint and/or the chewing musculature. Myofascial pain syndrome (MPS) is defined as pain that originates from myofascial trigger points in skeletal muscle. It is prevalent in regional musculoskeletal pain syndromes, either alone or in combination with other pain generators like Temporomandibular disorders (TMD). The appropriate evaluation and management of myofascial pain is an important part of musculoskeletal rehabilitation, and regional axial and limb pain syndromes. Currently a multifactorial theory on MPDS has received a great support among the scientific community. Myofascial Pain is the most common form of TMD, affecting principally women in reproductive age. This article gives a deeper insight into the pathogenesis, diagnosis and management of MPDS which will help clinicians to better understand this perplexing and mystifying condition.
\end{abstract}

Keywords: craniomandibular, myofascial pain dysfunction syndrome, patients, temporomandibular joint, trigger points.

\section{Introduction}

Craniomandibular disorders (CMD) are a combination of symptoms that may include pain, tenderness and dysfunction of the temporomandibular joint, the mouth and the occlusal contacts, the cervical spine, and the muscles of mastication. Patients may present with local dentoalveolar pain; muscle pain; head, facial and neck pain; sounds during condylar movements; deviations and limitations of mandibular movements; altered occlusal relations; parafunctions and poor oral habits; and functional limitations of mastication. It is generally accepted that CMD has a multifactorial etiology. ${ }^{1}$ Any disorder in different parts of masticatory system particularly in muscles, temporomandibular joint and its related structures can lead to pain and dysfunction in temporomandibular joint ${ }^{2,3}$. MPDS (Myofascial Pain Dysfunction Syndrome) is the most common form of temporomandibular disorders. Previous studies have shown muscular involvement in $90 \%$ of cases ${ }^{4,5}$. MPDS is the most common cause of oro-facial chronic pains ${ }^{6,7}$. In fact, MPDS is a psychological disorder which involves the masticatory muscles and results in pain, limitation in jaw movement, joint noise, jaw deviation in closing and opening the mouth and sensitivity in touching one or more masticatory muscles or their tendons ${ }^{8}$. The main acceptable factors include occlusion disorders and psychological problems ${ }^{9,10}$.

Different aspects relating to its physiopathology, clinical presentation and treatment remain to be clarified $^{11,12}$. MPDS was attributed to the inflammation of fibrous tissues around the ligaments, tendons, muscles and periosteum of the stomatognathic system. But, now, this syndrome has been broadly defined as "dysfunction of the masticatory and associated muscles characterised by pain"13. But no signs of obvious pathogenesis causing the syndrome have been found, it relies significantly on the clinical examination. MPDS is a disorder characterized by pain and dysfunction of the masticatory and associated muscles. It is one of the most common causes of chronic musculoskeletal pain ${ }^{14}$. Painful areas referred to as trigger zones were described by Travell and Rinzler (1952), who pointed out the existence of syndromes associated with trigger areas within the muscles coupled with pain, spasm, tenderness and dysfunction.In 1969

Laskin proposed a revised theory of Schwartz's TMJ pain dysfunction syndrome. He was of the view that although mechanical factors related to occlusion may sometimes be the cause of this condition by producing muscular overextension or overcontraction, the role of muscle fatigue was the most frequently encountered cause of such spasm. ${ }^{14}$ MPDS has a lifetime prevalence of $85 \%$ in general popuation ${ }^{14}$. Several studies show that it is more common in females. It is most frequently seen in young unmarried females (married to unmarried ratio 1:2) and female to male ratio is 3:1. It occurs commonly between 15-35 years of age, begins in teenagers and progresses into middle ageThe incidence of MPDS can be as high as 54\% in women and $45 \%$ in men, although the prevalence of TrPs doesn't exceed $25 \% .{ }^{15}$ 


\section{Etiopathogenesis}

MPDS has a multifactorial etiology. Several authors maintain that inadequate dentitions and unsatisfactory occlusion are the most frequent causes of MPDS. Other investigators noted that hyper function may provoke myofascial pain and assert that TMJ disturbances are usually related to dysfunction of the masticatory muscles and/or emotional disturbances. ${ }^{16}$ MPDS are characterized by the presence of hypersensitive spots called trigger points (TrPs). Such a syndrome is a regional disorder concerning the muscle, its fascia or both, and is accompanied by pain in an affected area and/or a zone of reference, autonomous phenomena and malfunction of the affected muscle. The presence of MPDS in the maxillofacial area mainly concerns the masseter, temporalis, lateral and medial pterygoid muscles.

TrPs produce pain to any activating stimulus (direct or indirect trauma) and can provoke referred pain, referred tenderness, motor dysfunction, autonomic phenomena and hyperexcitability of the central nervous system. Myofascial pain is perceived as a dull, non-pulsating pain, which can vary from mild discomfort to incapacitating pain, both at rest and during activity; it is rarely symmetric and adopts a segmented distribution (non-dermatomal spinal segmentation pattern) ${ }^{16}$.

Muscle overload is thought to be the result of sustained or repetitive low-level muscle contractions, eccentric muscle contractions, and maximal or submaximal concentric muscle contractions ${ }^{17}$. Although muscle damage is not required for the development of TrP, there may be a disruption of the cell membrane, damage to the sarcoplasmic reticulum with a subsequent release of high amounts of calcium-ions, and disruption of cytoskeletal proteins, such as desmin, titin, and dystrophin ${ }^{17}$.

MPDS are assumed to be caused by a number of unclear factors which include ${ }^{18}$ :

- Occlusal disturbances

- Intracapsular disorders

- Emotional turmoil.

The presynaptic, synaptic and postsynaptic mechanisms of abnormal depolarization (i.e. excessive release of acetylcholine (Ach), defects of acetylcholinesterase and upregulation of nicotine Ach-receptor activity, respectively) have been proposed as the possible etiological mechanisms. A more recent school of thought, the psycho physiologic theory, holds psychological and muscle factors to be of greatest importance.

The unifying concept proposed by Lerman - holds that MPDS results from the interplay of an unbroken chain of etiological factors ${ }^{16}$. The psychologic factors leads to an overall increase in masticatory muscle activity, while other factors such as occlusal and anatomic lead to selective increase in muscle activity.

Fig 1: mechanism to show how pain occurs ${ }^{18}$

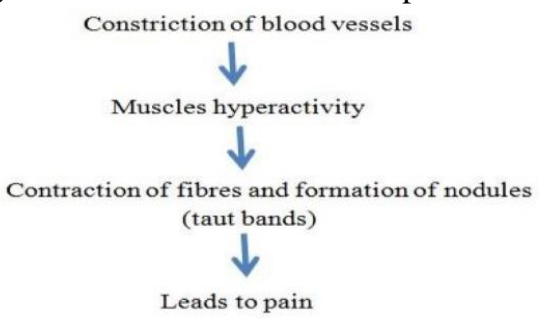

Fig 2: the below flowchart show some of the factors and how it leads to the myofascial pain dysfunction. ${ }^{18}$

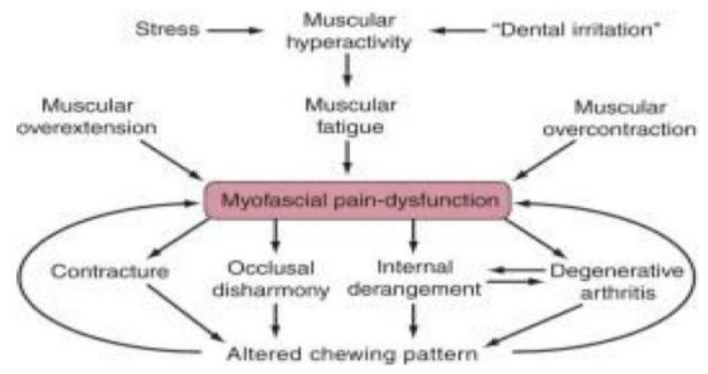

The development of trigger zone is considered as one of the most important characteristics features of MPDS.

Trigger Point ${ }^{18}$ One of the significant signs of MPDS is the presence of trigger points (TrP's) in a specific group of muscles.

"Trigger points are small exquisitely tender areas, which cause pain referred to a distant region, called the referred Pain Zone. They are activated by pressure, movement, change of barometric pressure and tension, be it 
physical or emotional."'Trigger points differ from "tender spots" (TS's) in the sense that the pain of TS's are localized in the surrounding of the spot while trigger points' pain refers to a distant area. However, the treatment of trigger points and TS's is exactly the same.

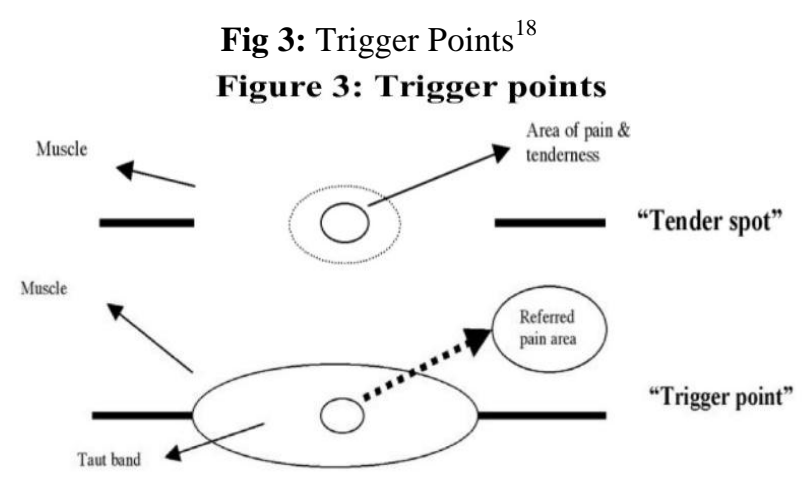

Trigger zone, is defined as a palpable focus of hyperirritability in a tissue that when compressed is locally tender and if sufficiently hypersensitive, gives rise to referred pain and tenderness.

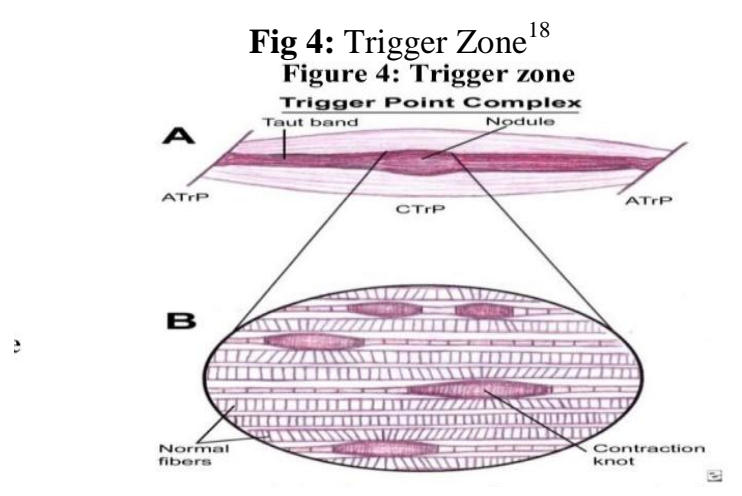

It is important to distinguish between myofascial pain and neuropathic pain. While myofascial pain originates at the muscle, neuropathic pain results from an injury to or malfunction of the peripheral or central nervous system ${ }^{13}$. There are myriad different pain syndromes and chronic pain disorder that fall into the category of neuropathic pain ${ }^{13}$. Myofascial pain, on the other hand, is thought to originate at the trigger point in the taut band of muscle.

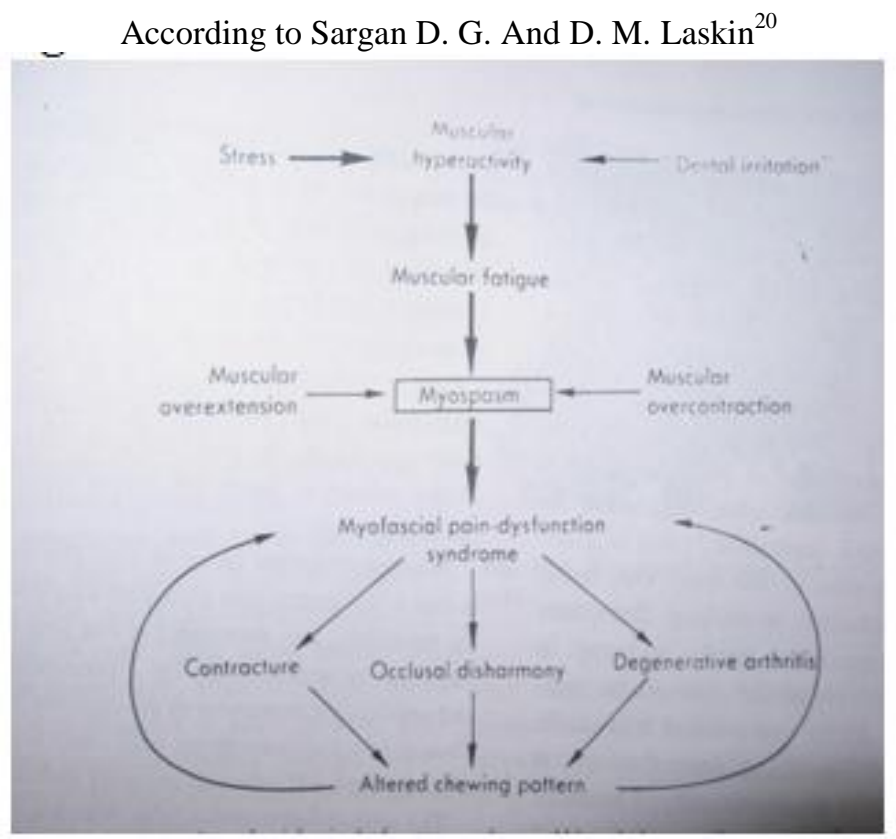

Fig 5 


\section{Pathophysiology}

1. Injury to muscle fiber type I

2. Metabolic distress at the motor end plates

3. Activation of muscle nociceptors

4. Transmission of pain to the CNS.

Knowing the potential causes of TrPs is important to prevent their development and recurrence, but also to inactivate and eliminate existing TrPs. There is general agreement that any kind of muscle overuse or direct trauma to the muscle can lead to the development of TrPs.

Muscle overload is thought to be the result of

- Sustained Low-Level Contractions and Intramuscular Pressure ${ }^{18}$

- Dynamic Concentric Contractions

- Cinderella Hypothesis

- Maximal or Submaximal Concentric Contractions ${ }^{18}$

- Eccentric Contractions ${ }^{18}$

- Modification of Central nociceptive input ${ }^{20}$

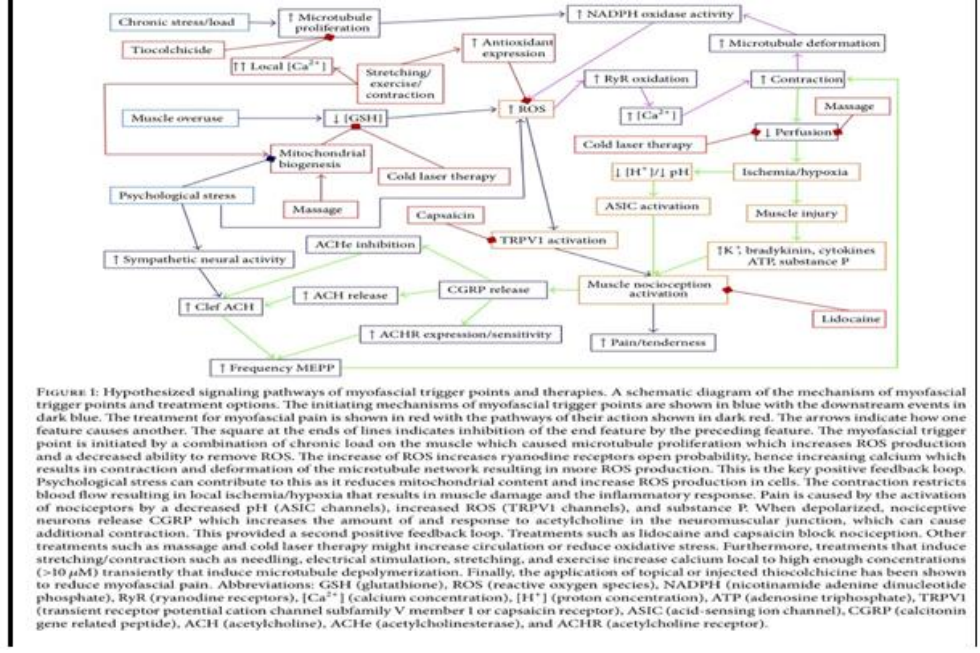

Fig 6

Initiation of Myofascial Trigger Points. Trigger points are thought to occur as a result of muscle overuse or muscle trauma or psychological stress ${ }^{21}$. Examples include trigger points arising secondary to muscle overload in worksite tasks or activities of daily living such as lifting heavy objects or sustained repetitive activities $^{21}$. Myofascial trigger points are more common under conditions of psychological stress. In fact, myofascial trigger points display increased myogenic activity, while the adjacent muscle remained silent under psychological stress ${ }^{21}$.

\section{Mechanisms for Persistence of Myofascial Trigger Points ${ }^{21}$.}

The persistence of myofascial trigger points requires a self- sustaining positive feed-forward process. Simons presented the integrated hypothesis for myofascial trigger points to offer an explanation ${ }^{21}$. The integrated hypothesis is a six- link chain that starts with step (1): the abnormal release of acetylcholine. This triggers step (2): increased muscle fiber tension which is seen as the taut band found in a myofascial trigger point. The taut band is thought to constrict blood flow that leads to step (3): local hypoxia. The reduced oxygen disrupts mitochondrial energy metabolism reducing ATP and leads to step (4): tissue distress and step (5): the release of sensitizing substances. These sensitizing substances lead to pain by activation of nociceptors (pain receptors) and also lead to step (6): autonomic modulation that then potentiates step (1): abnormal acetylcholine release.

To summarise

fig $7^{22}$ 


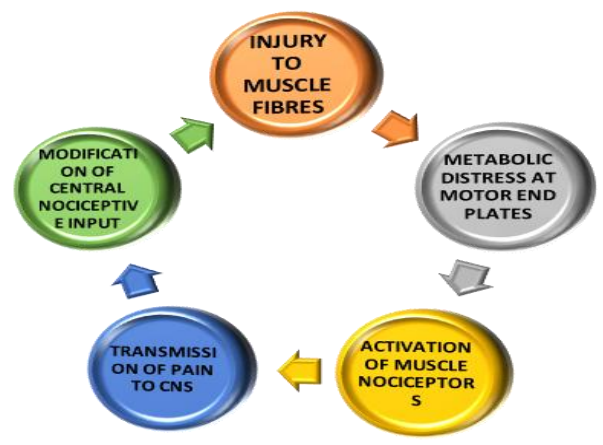

\section{Clinical Features}

MFP is a great imitator ${ }^{23}$. Myofascial pain tends to be dull, poorly localized, and deep, in contrast to the precise location of dental pain and cutaneous pain ${ }^{24}$. Clinically, referred pain is confusing to many clinicians, as frequently, patients complain more of pain in the referred pain zone and not necessarily of pain in the immediate area of a MTrP. Signs and symptoms suggestive of non-odontogenic pain include an inadequate local dental cause for the pain; a recurrence of pain in spite of reasonable dental therapy of the tooth or TMJ; poor lasting pain relief after local anesthetic blocking; positive findings with Rocabado's pain map; postural abnormalities such as forward head posture; and other pain problems, such as chronic and recurrent headaches and widespread chronic pain conditions ${ }^{24}$.

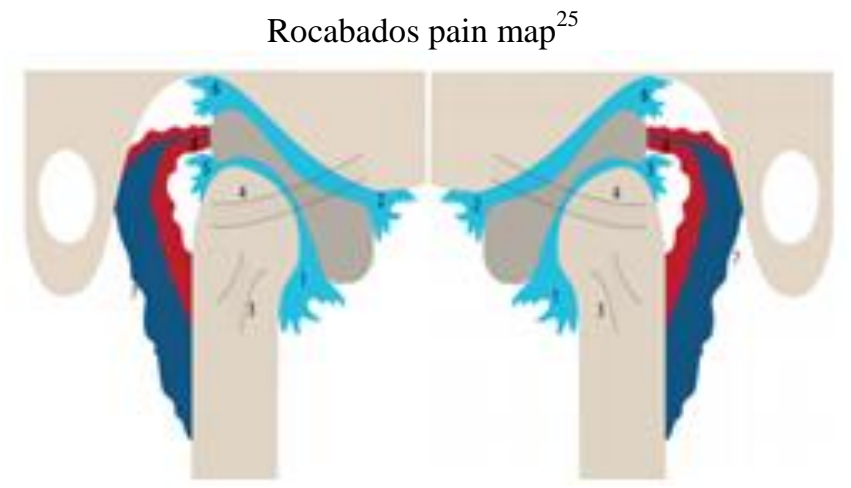

1. Anterior Inferior Synovial

2. Anterior Superior Synovial

3. Lateral Collateral Ligament

4. Temporomandibular Ligament

5. Posterior Inferior Synovial

6. Posterior Superior Synovial

7. Bilaminar Zone

8. Retrodiscal Tissue

Patients with MPS usually have a history of acute or chronic muscle overload. In dental practice, MPS is commonly seen in patients with a history of bruxism or clenching ${ }^{24}$. There are four cardinal signs and symptoms of the syndrome viz. pain, muscle tenderness, clicking or crepitus noise in the TMJ and unilaterally or bilaterally limitation of jaw movement with deviation on opening ${ }^{14}$.

Pain:

It can be either localized to the joint or referred to the head, neck or shoulders, it is usually unilateral and described as a dull ache in the ear or preauricular area which radiate to angle of the mandible, temporal area or lateral cervical area. Usually persistent in nature, often the pain is worse in the morning, in nocturnal bruxism or in the late afternoon if parafunctional clenching or bruxism are correlated with work stress.

\section{Tenderness:}

It is present over the affected TMJ during normal opening and closing motions. It is best elicited by placing the examining fingers at the posterosuperior aspect of both the condyles and expressing pressure 
anteriorly during excursion. It is more common over the condyle, above the maxillary tuberosity, at the angle of the mandible and the temporal crest.

\section{Clicking or popping noises in the tmj:}

They are common and described as clicking, popping or crepitus. The nature of the click is still uncertain. It is usually bilateral. It can occur at any point of jaw movement and there maybe multiple clicks. It may be audible, palpable or both and usually noted on simple palpation directly over the condylar head during the opening movement. Crepitus has been associated with perforations in the disk, which is usually followed by osteoarthritic change on the condylar surface followed by similar bony alterations on the opposing surface of the fossa.

\section{Deviation of the jaw to the affected side during normal opening motion:}

It is a common finding, since muscle spasm frequently accompanies joint dysfunction and as such contributes to the pain. This restricts the motion of the condyle, impairing or completely eliminating the forward gliding motion so that all that remains is a simple hinge action, with the condyle remaining in the fossa.

\section{Inability to close the posterior teeth completely into occlusion on the affected side: Discrepancy in occlusion may be due to ${ }^{14}$.}

A) Acquired malocclusion: the loss of the tooth without replacement will disrupt normal occlusion function by producing cuspal interference and premature contacts; resulting in alteration in joint function and the subsequent development of pain.

B) Inherent malocclusion: cuspal interference maybe considerable in a dentition in which no teeth have been lost, despite the fact that the teeth maybe acceptable cosmetically.

C) Improper dental restorations: may result in development of a painful TMJ. An important contributing factor is nervous tension with subsequent clenching, clamping or grinding of the teeth.

D) Nervous tension: it is an active factor in the production of joint pain. The clenching, clamping and grinding of the teeth are direct results of tension and produce a state of muscle fatigue that in itself maybe productive of pain even though the joint may not be involved. Pain and limitations of TMJ movement results from stress induced muscle contraction.

A diagnosis of MPDS can be made mainly by palpation. MPDS may actually be the most overlooked diagnosis in chronic pain patients ${ }^{24}$. The diagnosis depends on the clinician's skills, training and experience in taking a patient's history, performing a comprehensive examination, and assessing patients for $\mathrm{MTrPs}^{24}$. Simons, Travell and Simons defined a MTrP as a localized spot of tenderness in a nodule of a palpable taut band of contractured muscle fibers ${ }^{24}$. Taut bands are examined by gently palpating a muscle perpendicular to the direction of the muscle fibers. Taut bands need to be differentiated from more generalized muscle spasms that can be defined as electromyographic activity as a result of increased neuromuscular tone of the entire muscle ${ }^{24}$.

The presence of a local twitch response, referred pain, and the electromyographic demonstration of endplate noise increase the certainty and specificity of the diagnosis.

Local Twitch Response

A local twitch response (LTR) is elicited by either strong digital palpation or needling/injection of a taut band or $\mathrm{MTrP}$, that results in an involuntary brief burst of motor action potentials propagated only by fibers of that taut band or by fibers of a taut band from another muscle ${ }^{24}$.

\section{Referred Pain}

By mechanically stimulating an active MTrP, patients often report the development of referred pain in fairly consistent patterns, either immediately or after a brief 10-15 second delay.

\section{Investigations}

Clinical features are the commonest way to diagnose MPDS. Other investigations include radiological imaging $^{23}$. Imaging Studies: Imaging studies may provide useful anatomical information. However, MFP, TB, TRP and TS usually do not show up in these investigations ${ }^{23}$.

As it may be realised there is no distinctive radiographic feature of the MPDS ${ }^{22}$. There is a great deal of emphasis on the "joint space", however concentricity of the condyle in its fossa is quiet variable. When it appears displaced on radiographic examination such as tomography, arthrography, magnetic resonance imaging (MRI) the diagnosis is usually related to a disc displacement problem, and the clinical features are more consistent with that diagnosis than with MPDS $^{22}$.

Several plain film techniques are useful in these cases, including:

1. Transcranial

2. Transpharyngeal 
3. Transorbital

4. Panaromic

5. Specialised Panaromic

6. Tomography

Ultrasonography has proven to be useful in studying the condition of the muscles. Muscle activity can be investigated by electromyography.

\section{Management of mpds}

The management of MPDS includes pharmacological and non pharmacological methods followed by surgical methods being the last resort as follows:

Pharmacologic treatment includes use of various drugs to reduce the pain and discomfort caused to the patient.

Analgesic Drugs - Nonsteroidal anti-inflammatory drugs (NSAIDs)

Cyclooxygenase-2 (COX-2) inhibitors

Tramadol an opioid pain medication

Tropisetron a serotonin $5-\mathrm{HT}_{3}$ receptor antagonist

Muscle Relaxants -Tizanidine, Cyclobenzaprine

Anticonvulsants - Gabapentin

Antidepressants - Tricyclic antidepressants

\section{Nonpharmacologic Treatment Of Myofascial Pain}

Injections into MTrPs are a common and effective treatment, presumably due to mechanical disruption by the needle and termination of the dysfunctional activity of involved motor endplates ${ }^{26}$. MTrP injections may employ dry needling, short- or long-acting anesthetics, or steroids. Dry needling has been traditionally used as one of the fastest and most-effective ways to inactivate MTrPs and help alleviate the accompanied pain ${ }^{26}$.

Steroid injections into MTrPs are controversial and without clear rationale because little evidence exists to support an underlying inflammatory pathophysiology ${ }^{26}$. There are various sources in the literature, which have specifically described effective modalities, including deep-pressure massage ${ }^{29}$, stretch therapy with spray (where a taut band is stretched immediately after cold spray), superficial heat, and myofascial release ${ }^{26}$.

Ultrasound is a technique that has been proposed to treat myofascial pain by converting electrical energy to sound waves in order to provide heat energy to muscles ${ }^{26}$. Treatment of MPDS using ultrasonography combined with massage and exercise was performed by Dr Arme Gam and Susan Warming ${ }^{27}$.

Transcutaneous electric nerve stimulation (TENS) is a treatment modality that utilizes an electrical current to stimulate nerve fibers in order to provide pain relief ${ }^{26}$.

Magnetic stimulation $(M S)$ is a newer treatment that is being investigated for MSK pain and MPS. Only a limited number of studies exist and the exact therapeutic mechanism of action remains uncertain ${ }^{26}$.

Laser therapy has been used in the treatment of MSK pain including MPS; however, its exact mechanism of therapeutic action remains elusive.

\section{Exercise}

After needling the TRP/TS, it is essential to correct the muscle imbalance to achieve a good therapeutic result ${ }^{23}$. It is important to try to restore normal length and flexibility to the muscles. The following neuromuscular relaxation techniques may be applied ${ }^{23}$ :

i. Muscle relaxation by exhalation.

ii. Muscle relaxation by eye movement, inferiorly and in the direction in which relaxation is desired.

iii. Muscle relaxation following isometric contraction, byautogenic inhibition.

iv. Muscle relaxation following minimal activation of the antagonist muscle, by reciprocal inhibition.

Therapy for relief of dysfunction include restrictive use ${ }^{19}$, bio-feedback therapy: it gives patient voluntary control over automatically regulated body functions ${ }^{19}$, Occlusal splintsand nightguards ${ }^{19}$

Surgical management ${ }^{19}$ :

Surgical management should be considered only as a last resort. They are listed as follows:

Condylotomy

High condylectomy

Menisectomy

Myotomy.

Arthroscopy

Botulinum toxin $\mathrm{A}(\mathrm{BtA})$ injections: This method has been used successfully to treat both excessive clenching and recurrent TMJ dislocation ${ }^{19}$. 


\section{Conclusion}

Musculoskeletal pain is a major cause of morbidity. Its prevalence increases with age. A growing number of individuals in our ageing population have musculoskeletal pain that affects their daily activities and function ${ }^{14}$. It has a significant impact on their quality of life ${ }^{14}$. If they are left undiagnosed and untreated, it may develop into chronic pain with overlying psychosocial and functional problems ${ }^{23}$. This may lead to further distress, anxiety and eve depression. The viscous cycle may give rise to somatic preoccupation. This major source of musculoskeletal dysfunction requires more focused attention ${ }^{23}$. Its early diagnosis and treatment may help to reduce overlying psychosocial complications and the attending financial burden of chronic pain syndrome ${ }^{23}$.

Myofascial pain syndrome as defined by Simons, Travell, and Simons offers clinicians an excellent entry point into patients acute and chronic pain problems ${ }^{24}$. Myofascial trigger points are a common source or contributing factor to many musculoskeletal disorders. In the craniomandibular region, myofascial trigger points are frequently responsible for local and referred pain patterns, causing headaches, TMJ pain and dysfunction, tooth aches and facial pains ${ }^{24}$. Clinicans should routinely incorporate myofascial trigger points in their examinations and differential diagnosis ${ }^{24}$.It is essential that the correct diagnosis be made before treating MPDS. It is not a case of merely injecting trigger points and tender spots and hoping for the best. MPDS is self limiting if etiological factors are removed ${ }^{19}$.

Recent progress in experimental studies has provided a wealth of information that can be used to gain understanding of the syndrome ${ }^{21}$. Only through improved understanding of the molecular and subcellular pathways behind this disorder can novel therapeutics be discovered. Many details of the signaling pathways involved remain yet unclear and further study is needed ${ }^{28}$. Signs and symptoms related to temporomandibular disorders are various and are mostly time related, which is disappearance of some clinical manifestations of the disease and appearance of other ones later; so that the patient can experience different symptoms in every stage $^{43}$. Treatment of MPS finds that most interventions have demonstrated a limited body evidence for their use. This dearth of high quality evidence is due to the heterogeneity of MPS. The treatment for MPS should focus primarily on identifying and correcting the underlying cause of the symptoms. Surgical management should be considered only after reasonable nonsurgical efforts have been tried ${ }^{19}$.

Further research is needed to better establish algorithmic and evidence based treatment of MPS.

\section{References}

[1]. Curtis AW. Myofascial pain-dysfunction syndrome: the role of nonmasticatory muscles in 91 patients. Otolaryngol Head Neck Surg 1980;88(4):361-7

[2]. Laskin DM, Block S. Diagnosis and treatment of myofacial pain dysfunction (MPD) syndrome. J Prothest Dent 1986; 56(1): 7584.

[3]. Carlsson EG, Magnosson T. Management of temporomandibular disorders in the general dental practice. Chicago: Quintessence; 1999: 13-93.

[4]. Cardli P, Lattari M, Massaro P, Pllieita M. Pharmacologic treatment of the dysfunctional patient. J Minerva Stomatol 2005; 54(5): 265-79.

[5]. Rauhala K, Okarinen KS, Raustia AM. Role of temporomandibular disease in facial pain: Occlusion, muscle, and TMJ pain. J Cranio 1999; 17(4): 245-61.

[6]. Okeson JP. Management of temporomandibular disorders and occlusion. 1st ed. St Louis. Mosby, 1989; 150-86

[7]. Rezaei-Nejad A. Orofacial pain from basic sciences to clinical management. Tehran: Shayan Nemoudar; $2004: 5-87$.

[8]. Sherman D. Nonpharmacologic approaches to management of myofacial pain. J Temp Mand Disorder 2001; 5: 421-31.

[9]. Dolatabadi M, Kalantar-Motamed MH, Taheritalesh K. Temporomandibular disorders in the general dental practice. Tehran: Shayan Nemoudar; 2003: 5-27.

[10]. Honarmand M, Javadzadeh A, Toofaniasl H, Madani AA. Frequency of psychiatric disorder in patients with myofacial pain dysfunction syndrome. Journal of Mashhad Dental School 2009; 33(1): 77-82.

[11]. Lavelle ED, Lavelle W, Smith HS. Myofascial trigger points. Anesthesiol Clin. 2007;25:841-51.

[12]. Okeson JP. Management of temporomandibular disorders and occlusion. 4th ed. St. Louis, Mosby; 1998.

[13]. Pooja Arora, Roma Goswami, Shrimant Raman, Pulkit Jain.The Enigma Of Myofascial Pain Dysfunction Syndrome. International Journal of Advances in Scientific Research. 2015 Vol 01 issue 01.

[14]. Dr. Arun Garg, Dr. Shivani Jain, Dr. Shipra Gupta, Dr. Vandana Gupta.Myofacial Pain Dysfunction Syndrome: An Overview. Journal of Heal Talk, July-August 2013, volume05, issue 06

[15]. Eduardo Vasquez Delgado, Jordi Cascos Romero, Cosme Gay Escoda. Myofascial Pain Syndrome Associated with trigger point: A literature review. (I): Epidemology, clinical treatment and etiopathogeny.Med Oral Patol Oral Cir Bucal. 2009 Oct 1;14 (10):e494-8.

[16]. Wright EF. Referred craniofacial pain patterns in patients with temporomandibular disorder. J Am Dent Assoc. 2000;131:130715 .

[17]. Manolopoulos L, Vlastarakos PV, Georgiou L, Giotakis I, Loizos A, Nikolopoulos TP. Myofascial pain syndromes in the maxillofascial area: A common but underdiagnosed cause of head and neck pain. International Journal of Oral and Maxillofacial Surg. 2008;37: 975-984

[18]. Carol Bron, Jan D. Dommerholt.Etiology of Myofascial Trigger Points. Curr Pain Headache Rep (2012) 16:439-444

[19]. Pooja Arora, Roma Goswami, Shrimant Raman, Pulkit Jain. The Enigma of Myofascial Pain Dysfunction Syndrome. International Journal of Advances in Scientific Research. 2015 Vol 01 issue 01.

[20]. Laskin DM, Block S. Diagnosis and treatment of myofascial pain dysfunction (MPD) syndrome. J Prosthest Dent 1986; 56(1): $75-84$. 
[21]. M Saleet Jafri. Mechanisms of Myofascial Pain. International Scholarly Research Notices Volume012014, Article ID 523924.

[22]. Carolina Roldán Barraza. Myofascial Pain: Etiological factors and therapeutical methods. A systematic literature review of the last thirteen years.aus Santiago, Chile 2013

[23]. Eng-Ching Yap.Myofascial Pain- An Overview. Ann Acad Med Singapore 2007;36:43-8

[24]. Jan Dommerholt, Myofascial Pain Syndrome in the Craniomandibular Region. Padros Serrat, E. (ed) Bases diagnosticas, terapeuticas y posturales del functionalismo craniofascial. 2006;564-581.

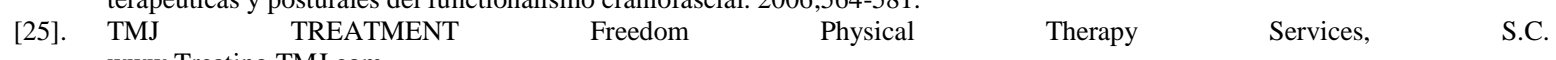
www.Treating TMJ.com

[26]. Mehul J. Desai, Vikramjeet Sainin, Shawnjeet Saini. Myofascial Pain Syndrome: A treatment Review. Pain Ther (2013) 2:21-36

[27]. Arne N Gam, Susan Warming, Lone Hørdum Larsen, Bente Jensen, Ola Høydalsmo, Ingelise Allon, Bente Andersen, Niels E Gøtzsche, Marelis Petersen, Birgitte Mathiesen. Treatment of myofascial trigger points with ultrasound combined with massage and exercise- a randomized control study. International Association for the Study of PainVolume 77, Issue 1, July 1998, Pages 73-79.

[28]. Hamed Mortazavi, Abbas Javadzadeh, Zahra Delavarian, Reza Zare Mahmoodabadi. Myofascial Pain Dysfunction Syndrome. Iranian Journal of Otorhinolaryngology vol. 22, No.61, Autumn-2010 